\title{
RESISTENCIA DE LOS PINOS A PLAGAS Y ENFERMEDADES: NUEVAS OPORTUNIDADES DE CONTROL FITOSANITARIO
}

\author{
Rafael Zas Arregui y Luis Sampedro Pérez \\ Misión Biológica de Galicia - Consejo Superior de Investigaciones Científicas. Grupo de Genética y \\ Ecología Forestal (www.genecolpines.weebly.es). Apdo. 28. 36080-PONTEVEDRA (España). Correo \\ electrónico: rzas@mbg.csic.es; lsampe@uvigo.es
}

\section{Resumen}

La lucha contra plagas y enfermedades forestales lleva preocupando a los selvicultores desde los comienzos de la gestión forestal. Tradicionalmente todo el esfuerzo se ha centrado en combatir al agente causante, buscando reducir las poblaciones o el impacto de éstas, ya sea mediante aplicación de productos químicos (insecticidas y fungicidas), captura directa con feromonas, medidas de saneamiento o las técnicas más recientes de control biológico. En general, salvo en el caso de insecticidas y fungicidas, las medidas de control no suelen ser totalmente eficaces, y sólo la combinación de múltiples métodos, por lo general costosos y difíciles de aplicar, consigue reducir de forma significativa el problema. A su vez, las restricciones ambientales, operativas y económicas inherentes a la gestión forestal, limitan de forma drástica la posibilidad de aplicación de productos fitosanitarios específicos y selectivos. En este contexto, y considerando el alarmante aumento de problemas sanitarios durante las últimas décadas en nuestras masas forestales, el estudio científico de los mecanismos de resistencia innatos de nuestras especies forestales a plagas y enfermedades adquiere una especial relevancia. Tras millones de generaciones, la convivencia con múltiples organismos nocivos ha desencadenado la evolución de complejos y eficaces mecanismos de resistencia. Aunque todavía poco estudiados en especies arbóreas por las dificultades asociadas a la investigación en organismos de vida larga y gran tamaño, hoy sabemos que la expresión de estos mecanismos de resistencia es extremadamente variable. Los mecanismos de resistencia dependen tanto de factores genéticos, como de factores ambientales abióticos y bióticos e incluso de complejas interacciones que involucran a múltiples componentes del ecosistema. Esta enorme variabilidad abre las puertas a la investigación de nuevas formas de gestión forestal sostenible orientada a maximizar la expresión de resistencia y tolerancia en las masas existentes o en las nuevas plantaciones forestales. En esta comunicación se ofrecen resultados recientes sobre las fuentes de variación de la resistencia a plagas y enfermedades en los pinos ibéricos, y el potencial existente para explotarlas en sanidad forestal.

Palabras clave: Defensas directas, Defensas indirectas, Resistencia constitutiva, Resistencia inducida, Tolerancia, Variación genética, Plasticidad fenotípica, Interacciones tritróficas, Compuestos orgánicos volátiles, Coníferas 


\section{INTRODUCCIÓN}

Los árboles, y en particular los pinos, llevan en la Tierra decenas de millones de años, y desde su aparición, conviven con multitud de organismos que utilizan los tejidos vegetales como fuente de recursos. Los insectos herbívoros y los hongos patógenos son los más frecuentes y dañinos. En esta larga historia de interacción entre huéspedes y hospedantes, ambos han desarrollado múltiples y variados mecanismos para optimizar su eficacia biológica en el contexto de la interacción. Como si de una batalla de armamento se tratara, los árboles han desarrollado una batería de defensas físicas y químicas para disuadir, evadir, repeler o minimizar el impacto de sus agresores bióticos. Por su parte, los insectos herbívoros y los hongos patógenos han generado mecanismos y habilidades para contrarrestar y superar estas barreras. A pesar de la clara desventaja evolutiva que supone la grandísima diferencia en el tiempo de generación entre huéspedes y hospedantes, los pinos han persistido a lo largo de las eras geológicas, y han dominado y dominan formando extensos bosques en gran parte de la superficie terrestre. No en vano, hoy en día el género Pinus, el más amplio de la familia Pinaceae, está representado por más de 100 especies que ocupan grandes superficies en las regiones de clima templado del hemisferio norte, en un amplio rango de condiciones ambientales, desde el subtrópico hasta las zonas boreales. Así, los pinos y sus enemigos llevan conviviendo en la tierra durante millones de años en un equilibrio dinámico, pero robusto. Con este pasado histórico, es lógico pensar que los pinos, como muchas otras especies de árboles y plantas, están dotados de unos mecanismos de resistencia extraordinariamente eficaces que han hecho posible su expansión y persistencia a lo largo de las eras geológicas.

A día de hoy, son varios los factores que están provocando un continuo deterioro sanitario de nuestras masas forestales y en particular de nuestros pinares (PELZ \& SMITH, 2012). El equilibrio natural entre los árboles y sus enemigos bióticos parece estar alterándose, y multitud de organismos nocivos progresan y avanzan causando graves y alarmantes daños en nuestras masas forestales. La tinta del castaño a principios del siglo pasado, la grafiosis de los olmos, los procesos de la seca de las quercíneas, las distintas plagas del eucalipto, o la reciente aparición del temido nematodo del pino, son buenos ejemplos de estos procesos de deterioro sanitario en las masas forestales de la Península Ibérica.

La progresión exponencial de la incidencia humana en el medio natural durante las últimas décadas o siglos está, probablemente, detrás de la mayoría, sino de todos, estos procesos de decaimiento forestal. Aunque no es fácil encontrar un denominador común, quizás el factor más relevante que explica esta proliferación de riesgos sanitarios es la expansión antrópica de plagas y enfermedades a nuevas regiones traspasando fronteras biogeográficas antes infranqueables (SANTINI et al., 2013). El fulminante desarrollo de la actividad humana durante los últimos años, $\mathrm{y}$ en particular el aumento galopante del transporte de mercancías a lo largo de todo el globo ha favorecido la expansión involuntaria de hongos e insectos entre biorregiones y continentes. Estos nuevos enemigos alóctonos, con los que la vegetación nativa no comparte un pasado evolutivo y por tanto contra los que no ha desarrollado mecanismos de resistencia específica, pueden llegar a mostrar un comportamiento invasivo y expandirse rápidamente causando graves daños en las masas locales. Otros factores como el cambio climático también favorecen la migración y expansión de plagas y enfermedades a nuevas zonas, alterando de nuevo el equilibrio entre los árboles y sus enemigos naturales. Desajustes fenológicos asociados al cambio climático también pueden favorecer la aparición y proliferación de nuevas amenazas. Por último, la alteración de la estructura genética intraespecífica de nuestras masas forestales a través de la introducción artificial de orígenes genéticos alóctonos o de material genéticamente mejorado contribuye a romper el equilibrio y también puede favorecer la progresión de plagas y enfermedades forestales. En el futuro próximo, los resultados aún desconocidos de la interacción entre todos estos factores podría incluso agravar los problemas.

\section{INTERACCIONES PLANTA-AGRESOR}

La lucha contra plagas y enfermedades forestales lleva preocupando a los gestores 
desde los comienzos de la gestión forestal. Tradicionalmente todo el esfuerzo se ha centrado en combatir al agente causante, buscando reducir las poblaciones o el impacto de éstas, ya sea mediante aplicación de productos químicos (insecticidas y fungicidas), captura directa con feromonas, medidas de saneamiento o las técnicas más recientes de control biológico. En general, salvo en el caso de insecticidas y fungicidas, las medidas de control no suelen ser totalmente eficaces, y sólo la combinación de múltiples métodos, por lo general costosos y difíciles de aplicar, consigue reducir de forma significativa el problema. A su vez, las restricciones ambientales, operativas y económicas inherentes a la gestión forestal, limitan de forma drástica la posibilidad de aplicación de productos fitosanitarios específicos y selectivos. En este contexto, y considerando el mencionado aumento de riesgos bióticos en nuestras masas, el estudio científico de los mecanismos de resistencia innatos de nuestras especies forestales a plagas y enfermedades adquiere una especial relevancia. Tras millones de generaciones, la convivencia con múltiples organismos nocivos ha desencadenado la evolución de complejos y eficaces mecanismos de resistencia que, como veremos en esta comunicación, muestran una enorme variación fenotípica. Esta variación intrínseca abre las puertas a nuevas oportunidades de control fitosanitario basadas en el fomento de la expresión de resistencia en las masas existentes o en las nuevas plantaciones forestales.

Durante las últimas décadas hemos sido testigo de formidables avances en el conocimiento del reino vegetal. El progreso en la comprensión de las interacciones de las plantas con los herbívoros y patógenos ha sido tal que está modificando la manera en la que vemos a los vegetales como organismos vivos. Así, estamos pasando de considerar a las plantas como organismos sésiles, pasivos y prácticamente inactivos, a asumir que las plantas son capaces de percibir el ambiente donde viven y modificar el fenotipo en consecuencia, que son capaces de identificar, reconocer y codificar estímulos bióticos, que reaccionan a éstos mediante la activación de rutas metabólicas plásticas, que estas reacciones son rápidas, sorprendentes, específicas, y en muchas ocasiones extremadamente eficaces, que las respuestas plásticas involucran cambios en la emisión de compuestos orgánicos volátiles que pueden modificar las interrelaciones de las plantas con otros miembros del ecosistema, o incluso con plantas vecinas, que las interacciones con organismos simbiontes de la rizosfera modula las interacciones bióticas de la parte aérea, y viceversa, etc., etc., etc.

Este complejísimo escenario de interacciones biológicas, donde las plantas son un recurso de comida plástico pero a la vez un nodo de información en el ecosistema, permite la aparición de nuevos y emergentes conceptos como comportamiento vegetal, inmunidad vegetal o comunicación entre plantas. Gracias al enorme progreso en las técnicas de biología molecular, día a día vamos desentrañando las bases moleculares de todas estas fascinantes propiedades de las plantas. A su vez, los veloces avances en el conocimiento básico de todos estos procesos derivan en nuevas y revolucionaras aplicaciones prácticas que empiezan a integrarse en la lucha contra plagas y enfermedades en el mundo agrícola. Un atractivo ejemplo de estas aplicaciones prácticas que integran los avances en el conocimiento de las interacciones bióticas para mejorar la productividad y sanidad de los cultivos son las técnicas "push-pull" desarrolladas en la Estación Rothamsted Research (KHAN et al., 2010). Intercalando en los cultivos plantas con efectos alelopáticos para plantas parásitas, plantas atrayentes de los principales herbívoros y sus parasitoides, así como con plantas cuyos volátiles repelen a ciertas plagas, estos investigadores consiguen aumentar la productividad del maíz y otros cultivos tropicales, de forma sostenible y respetuosa con el medio ambiente.

Sin embargo, no podemos olvidarnos de que todos estos avances, o la gran mayoría, se han realizado en unas pocas plantas modelo, herbáceas y anuales. Probablemente muchos de estos descubrimientos son extensibles a todo el reino vegetal pero todavía queda mucho camino que recorrer para aplicar este progreso a otros cultivos de interés agrario, y en particular, al mundo forestal. Probablemente por la dificultad asociada a la investigación con especies de ciclo de vida larga y de gran tamaño, los avances en el conocimiento de las interacciones bióticas con árboles son, todavía, muy reducidos. Sin embargo, la teoría predice que 
las estrategias plásticas, como la resistencia inducida, deberían estar favorecidas en especies longevas como los pinos, particularmente expuestas a la herbivoría y a los hongos patógenos. Además, la gran variabilidad intraespecífica existente en buena parte de nuestras especies de pino favorece las posibilidades de explotación.

A continuación se ofrece una pequeña revisión sobre los mecanismos de resistencia a enfermedades y plagas en los pinos ibéricos, describiendo las principales fuentes de variación fenotípica. Esta variación es el combustible para poner en marcha nuevas alternativas de control sanitario basadas en favorecer la expresión de la resistencia intrínseca que nuestras poblaciones de árboles han adquirido tras millones de años de evolución.

\section{DEFENSAS QUÍMICAS Y FÍSICAS EN CONÍFERAS}

Como en general en todo el reino vegetal, los mecanismos de resistencia a enfermedades y plagas en los pinos incluyen caracteres de defensa físicos que impiden o dificultan la penetración, consumo o degradación por parte de los organismos invasores, y compuestos químicos que resultan repelentes o tóxicos para éstos. Entre las primeras cabe citar la corteza, la presencia de espinas, pelos o tricomas, o la impregnación de distintos tejidos con polímeros resistentes a la degradación como la lignina o la suberina. Entre las segundas, las más importantes y estudiadas en coníferas son los compuestos carbonados de las familias de los terpenos y fenoles (FRANCESCHI et al., 2005; MOREIRA, 2010).

Sin duda, la defensa química más característica de los pinos es la producción de oleorresina, una substancia viscosa de propiedades defensivas variadas, producida a lo largo de todos los tejidos del árbol y principalmente en células especializadas que rodean los canales resiníferos (TRAPP \& CRoteAu, 2001) (Figura 1). La oleorresina está compuesta por una mezcla de monoterpenos y sesquiterpenos (fracción volátil) y diterpenos o ácidos resiníferos (fracción no volátil). Ante un daño producido por un agente agresor, la secreción de resina genera una importante barrera física. Pero, además, los terpenos que contiene resultan tóxicos para muchos insectos herbívoros y hongos patógenos, reduciendo, con ello, la proliferación del daño. Por último, la resina actúa como un eficaz sistema de sellado de las heridas producidas, impidiendo la infección por otros organismos y favoreciendo la recuperación tras el daño. Cuando los mono y sesquiterpenos se volatilizan en contacto con el aire, los diterpenos se oxidan y la resina remanente polimeriza generando una especie de costra en la herida. Por otra parte, la emisión de terpenos volátiles desde diferentes tejidos de la planta podría también contribuir a

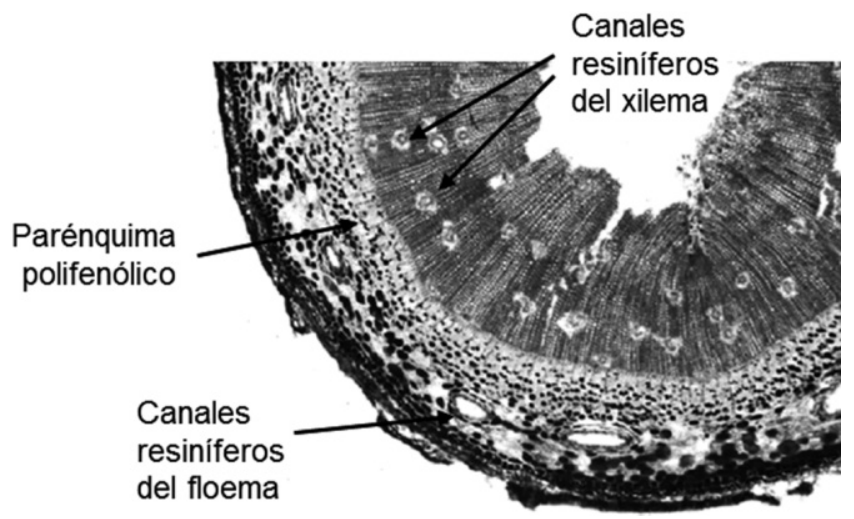

Figura 1. Corte transversal del tallo de una plántula de P. pinaster mostrando los canales resiníferos en el floema y en el xilema y el parénquima polifenólico. Los canales del floema conforman un sistema defensivo constitutivo especialmente sensible a las condiciones ambientales. Los canales del xilema aparecen de forma constitutiva pero también como respuesta a un ataque de un herbívoro en lo que se conoce como canales resiníferos traumáticos 
la resistencia de las plantas, por ejemplo, reduciendo su apariencia, al actuar como repelentes de ciertos herbívoros (MUMM \& HILKER, 2006).

Los compuestos fenólicos son el otro grupo mayoritario de compuestos químicos defensivos presentes en las coníferas. Aunque se conocen múltiples roles fisiológicos de esta gran familia de compuestos, sus propiedades tóxicas y repelentes para hongos e insectos son bien conocidas. Los fenoles están presentes principalmente en las acículas y en el floema y corteza, y su concentración es especialmente elevada en células especializadas del cortex que forman el parénquima polifenólico (FRANCESCHI et al., 2005).

Tanto la oleorresina como los compuestos fenólicos actúan como defensas químicas cuantitativas donde una mayor inversión, producción y acumulación de estas substancias se asocia con una reducción del daño causado por el agresor, es decir con una mayor resistencia al mismo. Con un efecto dosis-dependiente, la acumulación de estas substancias en los tejidos de los pinos llega a ser muy elevada, del orden de decenas de miligramos por gramo de tejido seco. Tratándose de compuestos carbonados, esto implica una gran inversión por parte de la planta que puede derivar en compromisos con otras funciones vitales como el crecimiento, la reproducción o la tolerancia a estreses abióticos.

Los mecanismos de defensa físicos y químicos resultan eficaces incrementando la resistencia frente a ataques de insectos y patógenos en la medida que son capaces de reducir los daños causados, bien sea evitando o reduciendo la incidencia del ataque (WAGNer et al., 2002). Sin embargo, los pinos, como la mayoría de las plantas y en especial las especies leñosas, también pueden presentar mecanismos de tolerancia mediante los cuales reducen el efecto negativo de los daños causados por el agresor sobre la eficacia biológica de la planta (STRAUSS \& AGRAWAL, 1999; ZAS et al., 2011). Este tipo de estrategia puede resultar especialmente relevante en especies longevas, de gran tamaño, y con reproducción retrasada, como los pinos. El aumento de la actividad fotosintética de las hojas remanentes, el incremento en la ramificación, la capacidad de retomar la dominancia apical tras la pérdida de la guía, la activación de meristemos durmientes, el almacenamiento y la redistribución de nutrientes inorgánicos y sustancias carbonadas son ejemplos de mecanismos de tolerancia mediante los cuales las plantas, y en particular los pinos, consiguen reducir el impacto del daño sobre el crecimiento y la reproducción (MoREIRA et al., 2012b; NúÑEZ-FARFÁN et $a l ., 2007)$.

\section{VARIACIÓN GENÉTICA}

Los pinos, como en general la mayoría de los árboles, muestran ciertas particularidades sorprendentes a nivel evolutivo. A pesar de que las tasas de mutación son más reducidas que en otros organismos, muestran una gran variabilidad genética intraespecífica (BusCHIAZZO et al., 2012). A su vez, aunque el flujo genético entre poblaciones es muy elevado, las poblaciones suelen estar fuertemente diferenciadas en caracteres adaptativos, a la vez que mantienen una elevada variación genética intrapoblacional (PetiT \& HAMPe, 2006). Entre otros, las elevadas tasas de flujo genético, la fuerte heterogeneidad ambiental dentro de las poblaciones tanto a nivel espacial, como a nivel temporal durante el largo ciclo de vida de los árboles, y los compromisos evolutivos en caracteres relacionados con la eficacia biológica ofitness, explican estas particularidades. La gran variabilidad genética existente dentro de poblaciones es la que permite obtener importantes ganancias en los programas de mejora genética forestal, a pesar de la sencillez de los procesos de mejora y de las enormes limitaciones que impone la longevidad y el retraso en la floración en especies arbóreas (ERIKSSON \& EKBERG, 2001). Así, con simples procesos de selección de árboles superiores dentro de una población y su posterior intercruzamiento, se obtienen, en una o en unas pocas generaciones, ganancias que compensan sobradamente las inversiones necesarias.

De igual manera que ocurre con muchos otros caracteres de historia vital, los caracteres de resistencia a enfermedades y plagas en los pinos siguen un patrón similar, con una alta diferenciación entre poblaciones y una gran variabilidad genética dentro de poblaciones. Bien porque la historia evolutiva de las distintas poblaciones en relación a la presión de enfermedades y plagas pudo ser muy diferente, o bien 
por otros factores relacionados con la diferenciación genética y ambiental entre poblaciones, en general las poblaciones de pino presentan altos niveles de diferenciación en relación a sus estrategias defensivas. Un conocido ejemplo de ello es la producción de resina en $P$. pinaster y la composición específica de terpenos que muestran claras diferencias a nivel de población (BARADAT et al., 1972). Esta diferenciación entre poblaciones también se ha observado en la resistencia efectiva a determinadas plagas, como por ejemplo a la cochinilla del pino, Matsococcus feytaudi, un insecto endémico en la zona oeste pero epidémico en el Sur de Francia, Italia y Córcega, zonas que ha colonizado recientemente $\mathrm{y}$ cuyas poblaciones de pino son especialmente susceptibles. A su vez, la variación genética de las poblaciones de este insecto, plaga específica del pino marítimo, guardan una estrecha relación con la estructuración genética de su único hospedante (HARFOUCHE et al., 1995) (Figura 2).

Los procesos de diferenciación genética suelen llevar asociados una reducción de la variabilidad genética interna dentro de las distintas poblaciones. Sin embargo, como ya se mencionó antes, las poblaciones de pino también muestran una gran variabilidad intrapoblacional en caracteres de historia vital, y los caracteres defensivos no son una excepción en ello. Existen varios ejemplos que indican variabilidad genética aditiva dentro de poblaciones en la inversión en compuestos defensivos (terpenos y fenoles) (MOREIRA et al., 2013; SAMPEDRO et al., 2010), así como variación genética en la resistencia efectiva a determinadas plagas y enfermedades. A modo de ejemplo, la población de mejora de $P$. pinaster de Galicia-Costa, población de variabilidad genética supuestamente reducida por los procesos de selección en base a un fenotipo común (buen crecimiento, rectitud del fuste, etc.), ha mostrado, sin embargo, variación genética aditiva en resistencia y/o tolerancia a distintas plagas y enfermedades, incluyendo el curculiónido comedor de floema y corteza Hylobius abietis (ZAS et al., 2005), la procesionaria del pino, Thaumathopoea pytiocampa, defoliador de acículas (DE LA MATA et al, no publicado), el hongo del suelo Armillaria ostoyae (Solla et al., 2011) y el temido hongo necrótico Fusarium circinatum (VIVAS et al., 2012). En general los niveles de heredabilidad individual son reducidos pero los coeficientes de variación aditiva son moderados o altos, posibilitando la mejora de la resistencia mediante procesos de selección clásica.

Curiosamente, a pesar de las enormes posibilidades que brinda esta gran variabilidad genética, la mejora de la resistencia a enfermedades y plagas mediante procesos de mejora genética ha sido, en general, poco utilizada en el mundo forestal. Pero no por ello deben menospreciarse las posibilidades existentes. Los éxitos obteni-

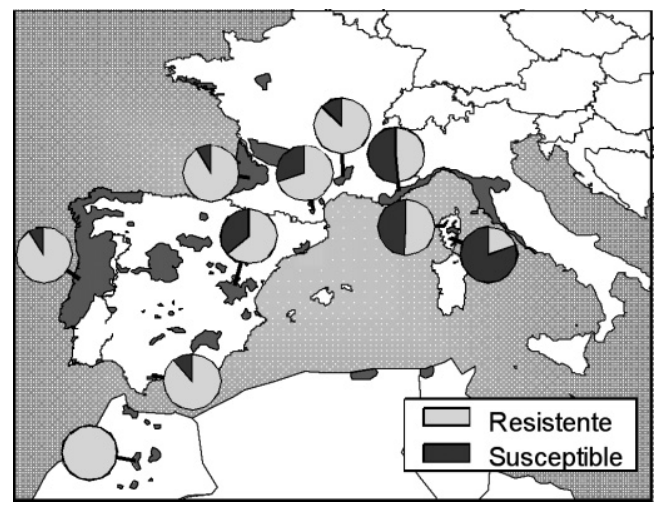

Figura 2. Diferenciación entre poblaciones de P. pinaster en resistencia a Matsucoccus feytaudi, una plaga específica de esta especie según evaluación en un ensayo de procedencias (Figura modificada de HaRFouCHE et al., 1995). Se observa un mayor porcentaje de individuos resistentes en las poblaciones del suroeste de la distribución natural del pino, donde esta plaga es endémica. Sin embargo, hacia el este, donde la plaga apareció recientemente, el nivel de resistencia es mucho menor 
dos en algunos programas como por ejemplo la mejora de la resistencia a Pissodes strobus en distintas especies de Picea de la costa oeste de Canadá (AlFARo et al., 2008), o la mejora de la resistencia al chancro del castaño en los castaños norteamericanos (ANAGNOSTAKIs, 2012), reflejan el potencial de estas técnicas. También en España hay buenos ejemplos que demuestran las posibilidades de mejora de la resistencia aprovechando la variabilidad genética existente en nuestras poblaciones. El caso del olmo, con varios genotipos resistentes a la grafiosis recientemente caracterizados (Solla et $a l$., 2005), o el caso de los híbridos de castaño para tolerar la tinta, son claros ejemplos exitosos. Otros programas de mejora avanzados como los programas de mejora genética de Pinus sylvestris y Picea abies de Suecia, están actualmente explorando las posibilidades de incluir aspectos relacionados con la resistencia a $H$. abietis, una de las plagas más problemáticas para la regeneración de coníferas en los países del Norte (Göran Nordlander, comunicación personal).

\section{VARIACIÓN AMBIENTAL}

Como especies longevas y sésiles, los pinos se caracterizan por una gran capacidad de acomodar su fenotipo a las condiciones ambientales particulares donde se desarrollan. Al igual que ocurre con otros caracteres de historia vital, la adjudicación de recursos a resistencia también muestra una gran plasticidad al medio abiótico, y en particular a la disponibilidad de recursos. La plasticidad en la adjudicación de recursos a resistencia en función de las condiciones ambientales se debe a dos motivos principales. Por un lado, la pérdida de tejidos por agresores bióticos tiene consecuencias mucho más críticas en situaciones limitantes que cuando los recursos abundan. Defender concienzudamente los tejidos puede ser la única estrategia viable en situaciones de fuerte estrés, ya que en caso contrario, los daños causados por los agresores podrían ser letales (STAMP, 2003). Por otro lado, el mantenimiento de los mecanismos de resistencia requiere la inversión de una gran cantidad de recursos, pero los recursos son limitados, y la adjudicación de recursos a resistencia puede estar comprometida con otras funciones vitales de la planta, como el crecimiento o la reproducción. El conflicto se resuelve según el balance entre costes y beneficios de la adjudicación a las distintas funciones. Pero este balance es contexto-dependiente resultando en una fuente de plasticidad en caracteres defensivos en función de las condiciones ambientales (KoRICHEVA, 2002).

Contrariamente a lo comúnmente pensando en el sector forestal, las distintas teorías predicen que la adjudicación relativa a resistencia es mayor cuanto menor sea la disponibilidad de recursos y peor sean las condiciones ambientales (e.g. STAMP, 2003). Así, muchas actividades de gestión que pretenden mejorar el rendimiento y el estado vegetativo de la masa, como por ejemplo la fertilización, pueden llevar asociadas un aumento de la susceptibilidad a los riesgos bióticos. Son muchos los trabajos científicos que han demostrado estas predicciones, encontrando una reducción de las defensas químicas cuando se aumenta la disponibilidad de recursos (nutrientes, luz, agua, $\mathrm{CO}_{2}$ ) (LOMBARDERO et al., 2000; LORIO et al., 2004; SAMPEDRO et $a l .$, 2011) (Figura 3a, 3b). Como ejemplo, cabe citar el claro aumento del consumo del curculiónido $H$. abietis en plantas fertilizadas y de mayor crecimiento de $P$. pinaster y $P$. radiata. La tasa de consumo fue hasta 3 veces mayor en las plantas fertilizadas, siendo el efecto especialmente patente cuando el tratamiento de fertilización incluía fósforo, el elemento más limitante en la zona de estudio, en su formulación (ZAS et al., 2006; ZAs et al., 2008) (Figura 3c). En este sentido, las técnicas de optimización de la fertilización en vivero, buscando un mayor crecimiento y mejor relación altura-diámetro, podrían no ser óptimas desde el punto de vista sanitario.

\section{PLASTICIDAD BIÓTICA}

Como ya hemos mencionado, el mantenimiento de estructuras defensivas físicas y químicas en las plantas supone un importante coste, coste que podría resultar en vano si finalmente la planta no es atacada. Dicho de otra manera, en ausencia de agresiones bióticas, una planta con pocas defensas constitutivas podría disponer de más recursos para crecer y reproducirse, y alcan- 

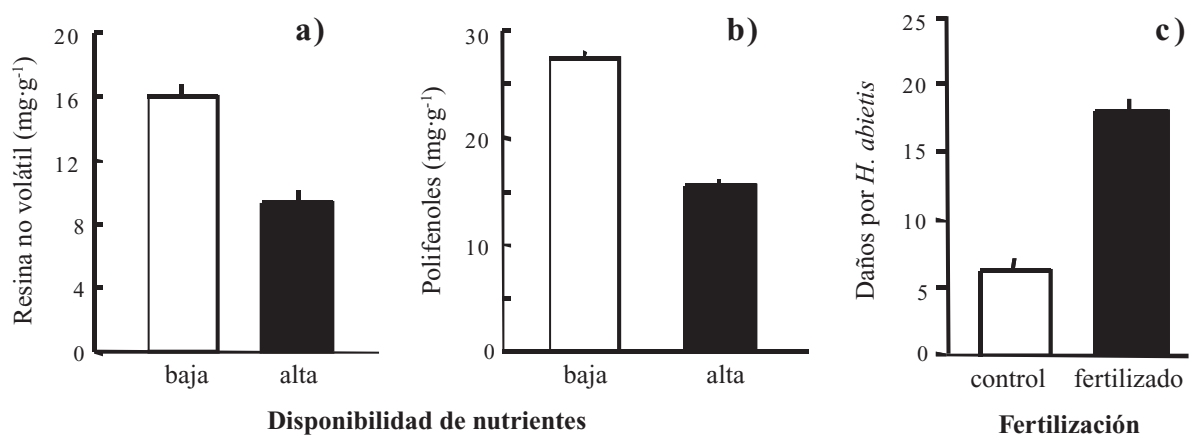

Figura 3. Efecto de la disponibilidad de nutrientes sobre la concentración constitutiva de compuestos defensivos en plántulas de P. pinaster cultivadas bajo condiciones controladas en invernadero (paneles a y b; datos de SAMPEDRO et al., 2011) y sobre el daño causado por el curculiónido H. abietis en un ensayo de P. pinaster en una zona de corta (panel $c$, datos de ZaS et al., 2006)

zar un mayor éxito biológico que una planta muy defendida. Estos costes han favorecido la evolución de la capacidad de modular la adjudicación de recursos a resistencia en función de la situación de riesgo biótico real, activando la producción de defensas tras la percepción de una agresión. Son las conocidas como defensas inducidas, o plasticidad biótica en resistencia. Aunque también costosas de producir, las defensas inducidas se consideran una estrategia ahorradora de recursos, ya que la adjudicación a resistencia sólo se realiza cuando es estrictamente necesaria, es decir tras un episodio de daño biótico (SAMPEDRO et al., 2011). Tras varias décadas de intensa investigación en esta fascinante capacidad de las plantas de responder a estímulos externos adaptando el fenotipo a la situación particular de riesgo, hoy en día, está bien asumido que la capacidad de expresar defensas inducidas es una estrategia general en el reino vegetal (HEIL, 2010; KARBAN, 2011), incluyendo por supuesto, a los pinos (EYLES et al., 2010).

La ventaja energética de las defensas inducidas se contrarresta con la desventaja funcional asociada al retraso temporal de la propia activación de las defensas. Entre el primer ataque y la activación de las defensas inducidas, las plantas permanecen vulnerables (HEIL \& BALDWIN, 2002). Por el contrario, las defensas constitutivas, presentes de forma basal y permanente, resultan eficaces porque están activas en el momento del ataque. Así, defensas constitutivas e inducidas actúan como un conjunto integrado en el espacio y en el tiempo. En primer lugar las defensas constitutivas repelen o inhiben el ataque. En caso de que esta barrera no sea suficiente, la activación de defensas inducidas contribuye a frenar la invasión y a proteger tejidos próximos todavía a salvo (FRANCESCHI et al., 2005).

Los sistemas de defensas inducidas en los pinos incluyen cambios cuantitativos y cualitativos en la química defensiva (terpenos y fenoles), así como cambios estructurales, como la diferenciación de canales resiníferos traumáticos, la proliferación de células polifenolicas parenquimáticas, o la generación de respuestas hipersensitivas (FRANCESCHI et al., 2005; MOREIRA, 2010). Algunas respuestas, como el aumento de la concentración y flujo de resina y cambios cualitativos en su composición son rápidas y ocurren a las horas o días de la primera agresión. Otros, como la producción de canales resiníferos traumáticos -canales de nueva formación que se forman en el xilema por una modificación de la actividad cambial, y que conforman una eficaz barrera a modo anillos concéntricosson más lentas y generan protección más a largo plazo. A su vez, las respuestas inducidas ocurren tanto en la proximidad de la zona de ataque como en zonas alejadas del mismo otorgando una mejora del nivel defensivo global de la planta, en lo que se denomina resistencia sistémica adquirida (DuRRANT \& DONG, 2004).

Que los pinos sean capaces de generar defensas inducidas implica que éstos son capaces de reconocer que están siendo agredidos, e 
incluso discriminar entre distintos tipos de agresores, así como enviar el mensaje de alerta a zonas meristemáticas para que éstas reaccionen y modifiquen su fisiología para aumentar su esfuerzo defensivo o modificar su patrón de diferenciación celular. En los últimos años se ha avanzado mucho en el conocimiento de las rutas metabólicas implicadas en la expresión de defensas inducidas en las que diversas fitohormonas juegan un papel esencial. Se ha descubierto también que la aplicación exógena de ciertas sustancias análogas a las fitohormonas implicadas en estas rutas induce la producción de defensas inducidas en las plantas de una manera semejante a la herbivoría real (Bohlmann, 2008). En particular, se sabe que el ácido jasmónico juega un papel muy importante en la señalización y activación de las defensas inducidas relacionadas con la herbivoría de insectos y hongos necrotrofos (MILLER et al., 2005), mientras que el ácido salicílico media las respuestas a las infecciones con hongos biotrofos e insectos chupadores de floema (PIETERSE et al., 2012; THALER et al., 2012). Como ocurre también en otras plantas, recientemente se ha demostrado en coníferas que la aplicación exógena de estas substancias, y en particular del metil jasmonato (MeJa) sobre la superficie de la corteza o fumigado sobre las hojas provoca respuestas equivalentes a las generadas por el ataque de organismos vivos (MOREIRA et al., 2011; MoreIRA et $a l ., 2012 \mathrm{c}$ ). Esto abre las puertas a una interesante y atractiva posibilidad de control sanitario basado en mejorar la resistencia de las plantas mediante la activación de su potencial de expresión de defensas. La posibilidad de regular la cantidad de esfuerzo que la planta dedica a defenderse mediante la simple fumigación de la parte aérea o la inclusión en el agua de riego de estas fitohormonas se presenta como una interesante herramienta de control sanitario, totalmente respetuosa con el medio ambiente. Este tipo de productos comienzan a utilizarse de forma comercial en agricultura, principalmente en agricultura biológica, pero todavía no han sido introducidos en el mundo forestal. Sin embargo, recientemente se ha comprobado que esta alternativa también puede resultar operativa y de gran utilidad para proteger plantas jóvenes de coníferas frente a plagas o enfermedades. Así, en un exhaustivo estudio realizado en Suecia y España, la fumigación en vivero con metil jasmonato se mostró efectiva reduciendo los daños generados por el curculiónido $H$. abietis una vez plantadas las plantas en monte. La aplicación de jasmonato redujo significativa y notablemente el nivel de consumo del insecto, reduciendo la mortalidad hasta niveles económicamente rentables (ZAs et al., 2013) (Figura 4). Estos resultados sugieren que la posibilidad de explotar la capacidad de las plantas de aumentar sus niveles defensivos mediante la simulación de ataques de herbívoros también puede resultar eficaz y operativa en el sector forestal.

\section{INTERACCIONES MULTITRÓFICAS}

Hasta aquí hemos visto que la expresión de mecanismos de resistencia constitutivos e inducidos muestra una gran variación fenotípica que está regulada tanto por factores genéticos como
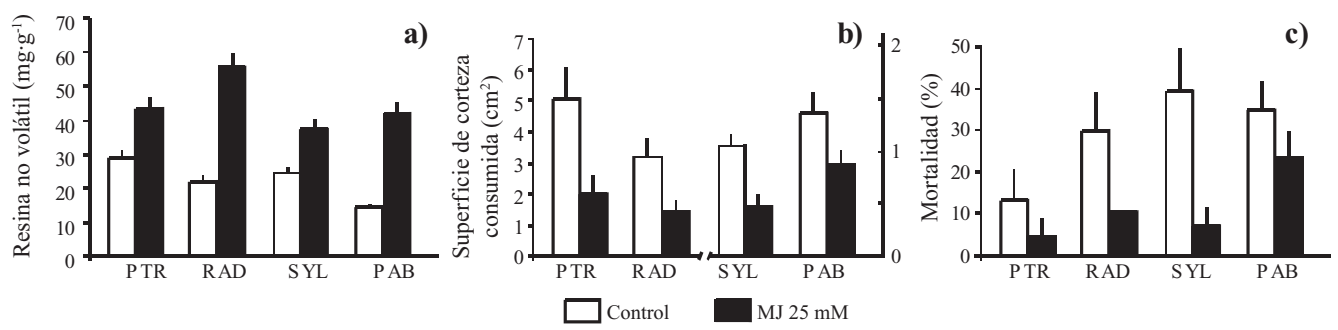

Figura 4. Respuesta de cuatro especies de coníferas (P. pinaster (PTR), P. radiata (RAD), P. sylvestris (SYL), P. abies $(P A B))$ a la aplicación exógena de meteil jasmonato $(M J)$ en vivero. a) Concentración de resina no volátil en el tallo, b) Superficie de corteza y floema consumida por el curculiónido H. abietis durante un período vegetativo en campo, c) Mortalidad debida al ataque del curculiónido tras dos períodos vegetativos (Datos de Zas et al., 2013) 
por factores ambientales abióticos y bióticos. Pero el escenario es todavía mucho más complejo existiendo muchos otros factores e interacciones que influyen en la capacidad de los pinos de hacer frente a sus enemigos naturales.

Tal como se trató previamente, las defensas físicas y químicas de los pinos son eficaces en la medida que reducen la incidencia del agente agresor sobre la planta. Esta es la forma estándar de actuación de los mecanismos de resistencia, donde la defensa en cuestión actúa de forma directa sobre la población del enemigo. Pero los mecanismos defensivos también pueden actuar de forma indirecta, a través de otros elementos bióticos del ecosistema. En su actividad diaria, las plantas mantienen un continuo intercambio gaseoso con la atmósfera, principalmente a través de los estomas. Además del intercambio de oxígeno y $\mathrm{CO}_{2}$ propio de la respiración y fotosíntesis, las plantas liberan compuestos orgáni$\cos$ volátiles a la atmósfera, que nosotros fácilmente percibimos e identificamos como el característico olor de un pinar o de un bosque de laureles, por poner dos ejemplos. Una respuesta inducida frecuente al ataque de insectos herbívoros y hongos patógenos es la alteración de la emisión de estos compuestos orgánicos volátiles (MOREIRA et al., 2013; SAMPEDRO et al., 2010). Estos cambios pueden ser percibidos por otros organismos del ecosistema, como predadores o parasitoides, que interpretan el mensaje químico como un aviso de una posible fuente de alimento o un sitio adecuado para realizar la puesta. Así, la alteración en la emisión de volátiles inducida por una agresión biótica sirve de defensa indirecta para la planta, donde el enemigo de su enemigo actúa en su beneficio. Los cambios en la emisión de volátiles pueden actuar, por tanto, como una señal química en complejas interacciones tritróficas (KESSLER \& BALDWIN, 2001).

Un buen ejemplo de cómo otros organismos pueden interferir en la capacidad de los pinos de combatir el ataque de una plaga es el caso de las hormigas que se alimentan de las secreciones azucaradas producidas por los áfidos. Los pinos albergan una gran comunidad de áfidos chupadores con los que conviven aparentemente sin demasiado problema. Estas colonias mantienen grandes colonias de hormigas que transitan de arriba abajo por los tallos desde los hormigueros hasta las fuentes de alimento. Como si de una granja se tratase, las hormigas mantienen a raya a cualquier intruso que se interponga en su camino y amenace a la colonia. Investigaciones recientes han demostrado que las plántulas con hormigas sufren menos daños por otros insectos herbívos, como el curculiónido H. abietis (MANÁK et al., 2013). El impacto de la gestión forestal sobre el suelo y los hormigueros debe por tanto ser considerado.

Otra fuente de variación fenotípica en aspectos relacionados con la resistencia de los pinos a sus enemigos naturales es la composición específica de la comunidad vegetal. Aunque parezca sorprendente, las interacciones bióticas entre los pinos y la comunidad de artrópodos que se desarrolla sobre ellos varía notablemente en función de la diversidad específica de la población donde habitan. Siguiendo con el ejemplo de los pinos, cuando los pinos crecen en monocultivos, con todos los individuos de una misma especie, las plántulas albergan comunidades de áfidos poco numerosas así como una baja densidad de hormigas asociadas a las colonias de áfidos. Sin embargo, cuando incrementamos la diversidad de especies de pinos en la plantacion, la colonización por áfidos y la de las hormigas que los atienden, aumenta hasta 3 veces más (MoREIRA et al., 2012a) (Figura 5). El aumento de la población de hormigas en las plantaciones pluriespecíficas se traslada en una reducción significativa de la comunidad de otros herbívoros dañinos, resultando en un mejor estado sanitario de las poblaciones mixtas respecto de las poblaciones monoespecíficas. Estos notables efectos se han observado mezclando especies del mismo género y relativamente próximas filogenéticamente y es posible que también ocurran a nivel intraespecífico, donde la diversidad genética de la población de una misma especie pueda influir significativamente en las interacciones con insectos herbívoros. A su vez, los resultados de este trabajo con plántulas de pino parecen extendibles a bosques maduros, donde se ha demostrado que los daños por herbivoría son menores en masas mixtas muy diversas que en masas monoespecíficas o con escasa diversidad específica (JACTEL \& BROCKERHOFF, 2007).

Por último, destacamos aquí otra fascinante fuente de variación fenotípica en aspectos defensivos relacionada con la emisión de volátiles por las plantas. Poco después de asumirse 

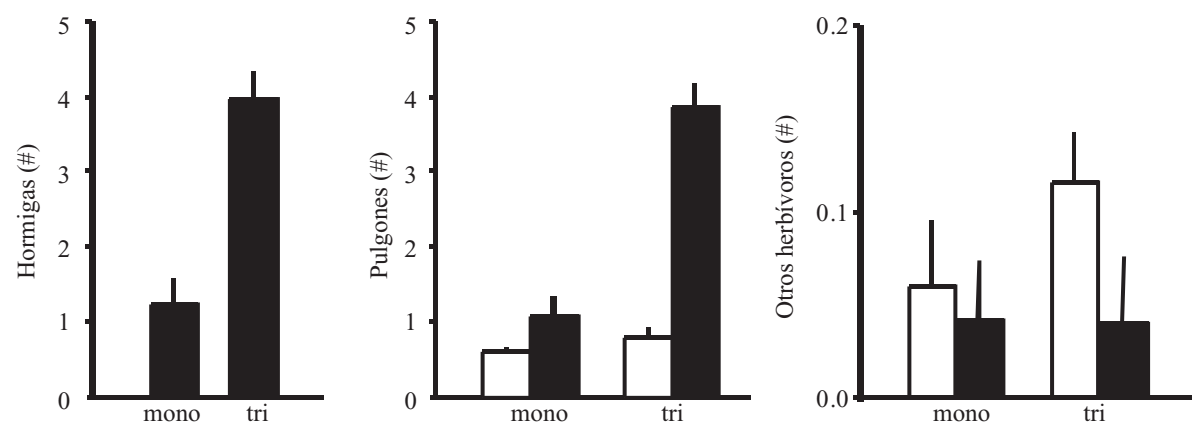

Diversidad específica (monocultivos vs tricultivos)
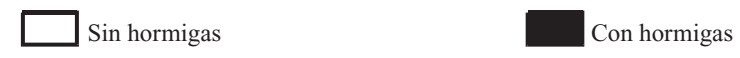

Figura 5. Influencia de la diversidad específica (monocultivos o mezclas de tres especies de pino) en la abundancia de hormigas, pulgones y otros herbívoros que albergan las plántulas de pino, restringiendo o no el acceso a los pinos de hormigas mutualistas (Datos tomados de MOREIRA et al., 2012a)

que los volátiles emitidos por las plantas jugaban un papel relevante en procesos de defensa indirecta, se barajó la posibilidad de que los volátiles emitidos pudieran también actuar como fuentes de información entre plantas. Si una planta no atacada es capaz de percibir la señal de alerta de una planta vecina atacada podría acomodar su fenotipo en consecuencia adelantándose a la inevitable agresión. Esto podría resultar en una clara ventaja adaptativa que reduciría los problemas derivados del retraso temporal de activación de las defensas inducidas. Así, la inversión en resistencia de una planta podría ajustarse en función de la percepción del riesgo real, pero con antelación a la materialización de la agresión. Los primeros estudios al respecto no fueron concluyentes y la comunidad científica no alcanzaba un consenso al respecto. Pero estudios más recientes, con metodologías depuradas y aproximaciones más finas, han demostrado que esta fascinante comunicación entre plantas realmente ocurre. Son ya muchos los trabajos que demuestran que determinadas especies de plantas son capaces de percibir y codificar las señales emitidas por plantas vecinas atacadas por herbívoros, reaccionando con respuestas defensivas directas o indirectas (HEIL \& Karban, 2010; Karban, 2008). Pero como suele ocurrir en estos campos de investigación frontera, los avances alcanzados hasta la fecha se centran sobre unas pocas especies anuales modelo, y la información existente en árboles es mucho más reducida (TSCHARNTKE et al., 2001).

En un trabajo preliminar realizado recientemente en la MBG-CSIC, hemos comprobado que los pinos también son capaces de percibir la herbivoría en su entorno y reaccionar activando respuestas inducidas. Plantas de $P$. radiata que convivieron en atmósferas aisladas con plantas de la misma especie inoculadas con el curculiónido $H$. abietis mostraron un aumento de la concentración de resina no volátil en la parte superior del tallo así como una alteración de la capacidad de respuesta a la herbivoría de este insecto (Diz, 2013). De forma similar, plantas de $P$. pinaster que crecieron en la proximidad de plantas atacadas por este insecto mostraron una mayor resistencia efectiva al insecto, siendo, al cabo del tiempo, menos consumidas (CASAS, 2015). En el video documental "El lenguaje de los bosques" se explican estas impresionantes y sorprendentes propiedades de los volátiles como medio de comunicación entre plantas (ZAS \& SAMPEDRO, 2012).

\section{Agradecimientos}

Este trabajo recoge resultados de experimentos realizados bajo distintos proyectos de investigación del Plan Nacional: FENOPIN (AGL2012-40151C03), COMPROPIN (AGL2010-18724), RTA2007-100, RTA2005-173 y PSE310000. 

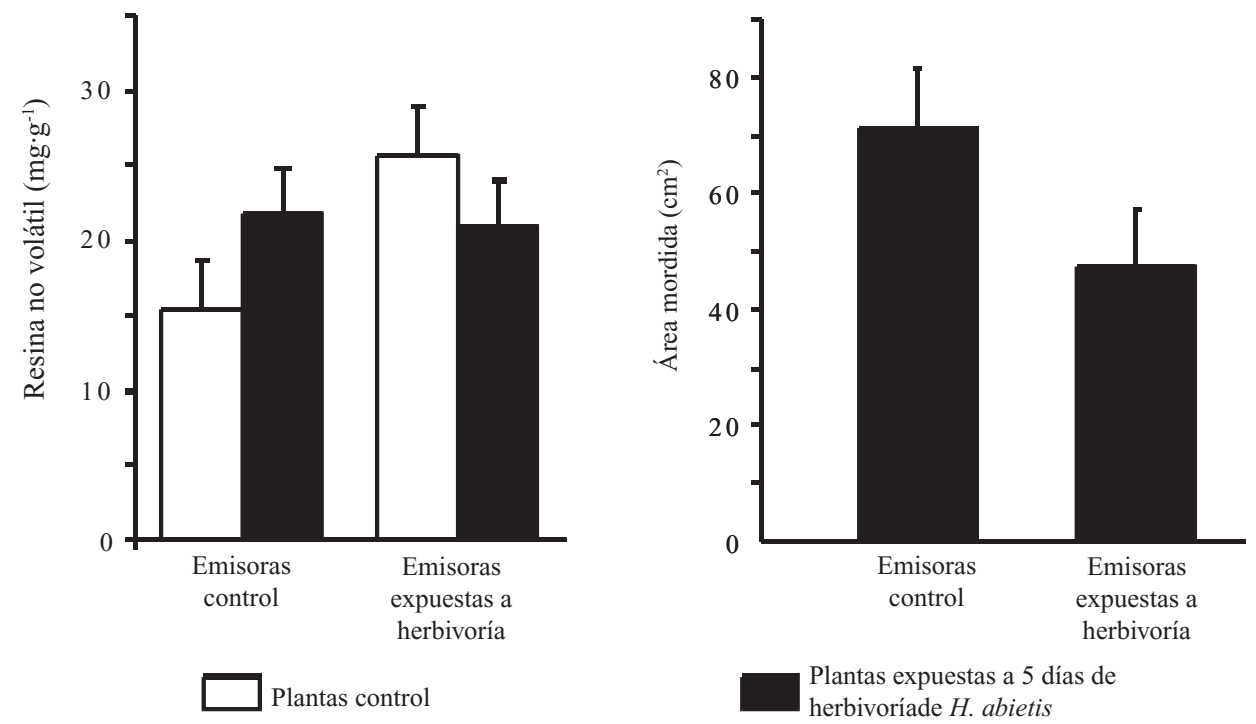

Figura 6. Influencia de la herbivoría de H. abietis en plantas vecinas sobre el contenido de resina no volátil en la parte superior del tallo de plántulas de P. radiata en respuesta a los daños causados por el curculiónido (a) y aumento de la resistencia efectiva frente a $\mathrm{H}$. abietis en plántulas de $P$. pinaster que crecieron junto a plantas de la misma especie atacadas por el curculiónido (b) (Datos tomados de: DIZ, 2013; CASAS, 2015)

Hasta 2013 LS ha disfrutado de un contrato posdoctoral INIA-CCAA en el Centro de Investigaciones Forestales de Lourizán. Los autores agradecen la fantástica labor realizada durante estos últimos años por los distintos miembros del grupo de investigación: Xoaquín Moreira, Vicente Rozas, Raúl de la Mata, César Cendán, Xosé Goldar, Benito Santos, Luz Pato, Rocío Campanó, entre otros, así como a los colaboradores Alejandro Solla, María Vivas, Elena Cubera, Beatriz Diz, Patricia Casas, Göran Nordlander y Niklas Björklund por sus contribuciones a la actividad del grupo.

\section{BIBLIOGRAFÍA}

Alfaro, R.I.; KInG, J.N.; Brown, R.G. \& BudDingH, S.M.; 2008. Screening of Sitka spruce genotypes for resistance to the white pine weevil using artificial infestations. Forest Ecol. Manage. 255: 1748-1758.

AnAgnostakis, S.L.; 2012. Chestnut Breeding in the United States for Disease and Insect Resistance. Plant Disease 96: 1392-1403.

Baradat, P.; Bernard, D.C.; Fillon, C.; Marpeau-bezard, A. \& PAuly, G.; 1972.
Les terpenes du pin maritime: aspects biologiques et genetiques. III Heredite de la teneur en monoterpenes. Ann. Sci. For. 29: 397-334.

BoHLMANN, J.; 2008. Insect-induced terpenoid defenses in spruce. In: A. Schaller (ed.), Induced plant resistance to herbivory: 173187. Springer Netherlands. Dordrecht.

Buschiazzo, E.; Ritland, C.; BohlmanN, J. \& RITLAND, K.; 2012. Slow but not low: genomic comparisons reveal slower evolutionary rate and higher $\mathrm{dN} / \mathrm{dS}$ in conifers compared to angiosperms. BMC Evolutionary Biology 12: 8.

CAsas, P.; 2015. Percepción del riesgo de la herbivoría en la vecindad en dos pinos de interés forestal: defensa efectiva frente a un insecto herbivoro. Proyecto Fin de Carrera. Escuela Técnica de Enxeñeiros de Montes. Universidade de Vigo. Pontevedra.

Diz, B.; 2013. Percepción do risco de herbivoría en dous piñeiros de interés forestal: cambios na química defensiva. Proyecto Fin de Carrera. Escuela Técnica de Enxeñeiros de Montes. Universidade de Vigo. Pontevedra.

Durrant, W.E. \& Dong, X.; 2004. Systemic acquired resistance. Annual Rev. Phytopathol. 42: 185-209. 
ERIKSSON, G. \& EKBERG, I.; 2001. An Introduction to Forest Genetics. Swedish University of Agricultural Sciences. Uppsala.

Eyles, A.; Bonello, P.; GANLEy, R. \& Mohammed, C.; 2010. Induced resistance to pests and pathogens in trees. New Phytologist 185: 893-908.

Franceschi, V.R.; KroKene, P. \& KREKLING, T.; 2005. Anatomical and chemical defenses of conifer bark against bark beetles and other pests. New Phytologist 167: 353-376.

Harfouche, A.; Baradat, P.; Durel, C.E. \& POMMERY, J.; 1995. Variabilité intraspécifique chez le pin maritime (Pinus pinaster Ait) dans le sud-est de la France. I. Variabilité des populations autochtones et des populations de l'ensemble de l'aire de l'espèce. Ann. Sci. For. 52: 307-328

HeIL, M.; 2010. Plastic defence expression in plants. Evolutionary Ecology 24: 555-569.

HeIL, M. \& Baldwin, I.; 2002. Fitness costs of induced resistance: emerging experimental support for a slippery concept. Trends in Plant Science 7: 61-67.

HeIL, M. \& Karban, R.; 2010. Explaining evolution of plant communication by airborne signals. Trends in Ecology and Evolution 25: 137-144.

Jactel, H. \& Brockerhoff, E.G.; 2007. Tree diversity reduces herbivory by forest insects. Ecology Letters 10: 835-848.

KARBAN, R.; 2008. Plant behaviour and communication. Ecology Letters 11: 727-739.

KARBAN, R.; 2011. The ecology and evolution of induced resistance against herbivores. Functional Ecology 25: 339-347.

Kessler, A. \& Baldwin, I.T.; 2001. Defensive function of herbivore-induced plant volatile emissions in nature. Science 291(5511): 2141-2144.

Khan, Z.R.; Midega, C.A.O.; Bruce, T.J.A. et $a l . ; 2010$. Exploiting phytochemicals for developing a 'push-pull' crop protection strategy for cereal farmers in Africa. J. Exp. Bot. 61: 4185-4196.

KoRICHEVA, J.; 2002. Meta-analysis of sources of variation in fitness costs of plant antiherbivore defenses. Ecology 83(1): 176-190.

Lombardero, M.J.; AYres, M.P.; LORIO, P.L. \& RUEL, J.J.; 2000. Environmental effects on constitutive and inducible resin defences of Pinus taeda. Ecology Letters 3: 329 -339

Lorio, J.P.L.; StePhen, F.M. \& PAINe, T.D.; 2004. Environment and ontogeny modify loblolly pine response to induced acute water deficits and bark beetle attack. Forest Ecol. Manage. 73: 97-110.

ManÁK, V.; NoRdenhem, H.; BJÖRKLUnd, N.; LENOIR, L. \& NoRDLANDER, G.; 2013. Ants protect conifer seedlings from feeding damage by the pine weevil Hylobius abietis. Agric. For. Entomol. 15: 98-105.

Miller, B.; Madilao, L.L.; RalPh, S. \& BOHLMANN, J.; 2005. Insect-induced conifer defense. White pine weevil and methyl jasmonate induce traumatic resinosis, de novo formed volatile emissions, and accumulation of terpenoid synthase and putative octadecanoid pathway transcripts in Sitka spruce. Plant Physiology 137: 369-382.

MoReIRA, X.; 2010. Variación genética y efecto de la disponibilidad de nutrientes en las defensas constitutivas e inducidas de Pinus pinaster Ait. $\mathrm{PhD}$ Dissertation. University of Vigo. Vigo.

Moreira, X.; KaIlen, A.M.; Zas, R. \& SAMPEDRO, L.; 2012a. Bottom-up effects of host-plant species diversity and top-down effects of ants interactively increases plant performance. Proceedings of the Royal Society B 279: 4464-4472.

Moreira, X.; SAMPEdro, L. \& ZAS, R. 2011. Methyl jasmonate as chemical elicitor of induced responses and anti-herbivory resistance in young conifer trees. In: J.M. Merillon, J.M. \& K.G. Ramawat (eds.), Plant defence: Biological control. Progress in Biological Control: 345-362. Springer Netherlands. Dordrecht.

Moreira, X.; Zas, R. \& SAMPEdRO, L.; 2012b. Genetic variation and phenotypic plasticity of nutrient re-allocation and increased fine root production as putative tolerance mechanisms inducible by methyl jasmonate in pine trees. J. Ecology 100: 810-820.

Moreira, X.; Zas, R. \& SAMPedro, L.; 2012c. Quantitative comparison of chemical, biological and mechanical induction of secondary compounds in Pinus pinaster seedlings. Trees Structure and Function 26(2): 677-685. 
Moreira, X.; Zas, R. \& SAMPedro, L.; 2013. Additive genetic variation in resistance traits of an exotic pine species: little evidence for constraints on evolution of resistance against native herbivores. Heredity 110: 449-456.

MuMm, R. \& HiLKeR, M.; 2006. Direct and indirect chemical defence of pine against folivorous insects Trends in Plant Science 11: 351-358.

NúÑEZ-FARFÁn, J.; Fornoni, J. \& VAlverde, P.L.; 2007. The evolution of resistance and tolerance to herbivores. Annual Rev. Ecol. Evol. Systematics 38: 541-566.

Pelz, K.A. \& Smith, F.W.; 2012. Thirty year change in lodgepole and lodgepole/mixed conifer forest structure following 1980s mountain pine beetle outbreak in western Colorado, USA. Forest Ecol. Manage. 280: 93-102.

Petit, R.J. \& Hampe, A.; 2006. Some evolutionary consequences of being a tree. Annual Rev. Ecol. Evol. Systematics 37: 187-214.

Pieterse, C.M.J.; VAN Der Does, D.; Zamioudis, C.; LeON-ReYes, A. \& VAN WeES, S.C.M.; 2012. Hormonal Modulation of Plant Immunity. Annual Rev. Cell Develop. Biol. 28: 489-521.

SAmpedro, L.; Moreira, X.; Llusia, J.; Peñuelas, J. \& Zas, R.; 2010. Genetics, phosphorus availability, and herbivore-derived induction as sources of phenotypic variation of leaf volatile terpenes in a pine species. J. Exp. Bot. 61: 4437-4447.

SAMPEDro, L.; MoreIra, X. \& Zas, R.; 2011. Costs of constitutive and jasmonate-induced pine tree chemical defences emerge only under low nutrient availability. J. Ecology 99: 818-827.

Santini, A.; Ghelardini, L.; De Pace, C. et-al.; 2013. Biogeographical patterns and determinants of invasion by forest pathogens in Europe. New Phytologist 197: 283-250.

Solla, A.; Aguín, O.; Cubera, E.; SAmpedro, L.; Mansilla, P. \& ZAS, R.; 2011. Survival time analysis of Pinus pinaster inoculated with Armillaria ostoyae: genetic variation and relevance of seed and root traits. Eur. $J$. Plant Pathol. 130: 477-488.

Solla, A.; Bohnens, J.; Collin, E.; Diamandis, S.; FranKe, A.; GIL, L.; BuRÓN, M.; SAntini, A.; Mittempergher, L.;
Pinon, J. \& Vanden Broeck, A.; 2005. Screening European Elms for Resistance to Ophiostoma novo-ulmi. Forest Science 51(2): 134-141.

STAMP, N.; 2003. Out of the quagmire of plant defense hypotheses. Quart. Rev. Biol. 78(1): 23-55.

Strauss, S.Y. \& Agrawal, A.A.; 1999. The ecology and evolution of plant tolerance to herbivory. Trends in Ecology \& Evolution 14(5): 179-185.

Thaler, J.S.; Humphrey, P.T. \& Whiteman, N.K.; 2012. Evolution of jasmonate and salicylate signal crosstalk. Trends in Plant Science 17: 260-270.

Trapp, S. \& CRoteau, R.; 2001. Defensive resin biosynthesis in conifers. Annual Rev. Plant Physiol. Plant Mol. Biol. 52: 689-724.

TscharntKe, T.; Thiessen, S.; Dolch, R. et $a l$;; 2001. Herbivory, induced resistance, and interplant signal transfer in Alnus glutinosa. Biochem. Systematics Ecol. 29: 1025-1047.

Vivas, M.; Zas, R. \& Solla, A.; 2012. First screening of a native European pine (Pinus pinaster) for resistance to Fusarium circinatum. Forestry 85: 185-192.

Wagner, M.R.; Clancy, K.M.; Lieutier, F. \& PAINE, T.D.; 2002. Mechanisms and deployment of resistance in trees to insects. Kluwer Academic Publishers. London.

Zas, R.; BJÖRKLund, N.; Nordlander, G.; Cendán, C.; Hellqvist, C. \& Sampedro, L.; 2013. Exploiting jasmonate-induced responses for field protection of conifer seedlings against a major forest pest, Hylobius abietis. Forest Ecol. Manage. 313: 212-223.

Zas, R.; Moreira, X. \& SAMPedro, L.; 2011. Tolerance and induced resistance in a native and an exotic pine species: relevant traits for invasion ecology. J. Ecology 99: 1316-1326.

ZAS, R. \& SAMPEDRO, L.; 2012. El lenguaje de los bosques. Video documental de divulgación científica. Disponible en internet en https://www.youtube.com/watch?v=K9KA9 F4JgQA

ZAS, R.; SAMPedro, L.; Moreira, X. \& MARTíns, P.; 2008. Effect of fertilization and genetic variation on susceptibility of Pinus radiata seedlings to Hylobius abietis damage. Can. J. For. Res. 38: 63-72. 
Zas, R.; SAmpedro, L.; Prada, E. \& FERNÁNDEZ-LÓPEZ, J.; 2005. Genetic variation of Pinus pinaster Ait. seedlings in susceptibility to Hylobius abietis L. Ann. For. Sci. 62: 681-688.
ZAS, R.; SAMPEDRO, L.; PRADA, E.; LOMBARDERO, M.J. \& FERNÁNDEZ-LÓPEZ, J.; 2006. Fertilization increases Hylobius abietis L. damage in Pinus pinaster Ait. seedlings. Forest Ecol. Manage. 222: 137-144. 